\title{
Generative Adversarial Networks Time Series Models to Forecast Medicine Daily Sales in Hospital
}

\author{
Amir Mahmud Husein \\ Universitas Prima Indonesia \\ Fakultas Teknologi dan Ilmu Komputer, Teknik \\ Informatika \\ Jl. Sekip Sei Sikambing Medan \\ amirmahmud@unprimdn.ac.id \\ Sutrisno Sinaga \\ Universitas Prima Indonesia \\ Fakultas Teknologi dan Ilmu Komputer, Teknik \\ Informatika \\ Jl. Sekip Sei Sikambing Medan \\ Trisnosinaga78@gmail.com
}

\author{
Muhammad Arsyal \\ Universitas Prima Indonesia \\ Fakultas Teknologi dan Ilmu Komputer, \\ Teknik Informatika \\ J1. Sekip Sei Sikambing Medan \\ Arsyalm280@gmail.com \\ Hendra Syahputa \\ Universitas Prima Indonesia \\ Fakultas Teknologi dan Ilmu Komputer, \\ Teknik Informatika \\ J1. Sekip Sei Sikambing Medan \\ Hendramanik100@gmail.com
}

\begin{abstract}
The success of the work of Generative Adversarial Networks (GAN) has recently achieved great success in many fields, such as stock market prediction, portfolio optimization, financial information processing and trading execution strategies, because the GAN model generates seemingly realistic data with models generator and discriminator .Planning for drug needs that are not optimal will have an impact on hospital services and economics, so it requires a reliable and accurate prediction model with the aim of minimizing the occurrence of shortages and excess stock, In this paper, we propose the GAN architecture to estimate the amount of drug sales in the next one week by using the drug usage data for the last four years (2015-2018) for training, while testing using data running in 2019 year, the classification results will be evaluated by Actual data uses indicators of Mean Absolute Error (MAE), Root Mean Square Error (RMSE) and Mean Absolute Percentage Error (MAPE). From the results of the experiment, seen from the value of MAE, RMSE and MAPE, the proposed model has promising performance, but it still needs to be developed to explore ways to extract factors that are more valuable and influential in the trend disease progression, thus helping in the selection of optimal drugs.
\end{abstract}

Keywords-Forecasting, Generative Adversarial Networks (GAN), deep learning, Stock Prediction;

\section{INTRODUCTION}

The implementation of electronic health records (EHRs) has an impact on improving health services that make it easier to get information when needed, such as prescription drug information used by doctors in patients. information on drug use can be used to develop prediction models drug needs as needed. Forecasting the need for number of drugs aims to avoid the occurrence of vacancies or excess drugs. In general, conventional modeling such as the consumption, morbidity and joint model proved to be accurate, but the uncertainty in the number of requests and the frequency of disease progression made this method less accurate [1] so that a prediction model based on population and patient disease was needed [2], time series are widely proposed by researchers in various fields such as financial prediction [3] [4], water quality [5], Hospital Management Inventory [6], Sales Prediction [7], Inventory [8], Backorder Material [9], food demand [10], Electricity Prices [11], [12], [13]. Model selector and forecasting accuracy is one of the important criteria in decision making [14]. Traditional prediction models 
such as Auto-Regressive Integrated Moving Average (ARIMA), regression, exponential smoothing, Kalman filtering, Bayesian estimation using historical data [15], besides in Machine Learning (ML) approach with random forest regression, quantile random forests and artificial neural networks (ANNs) have weaknesses in probabilistic estimates [16].

Recently deep learning for the task of classify time series is many proposed [17] with approaches to various models, such as Recurrent Neural Networks (RNN) [18], [19], Product-based Neural Networks (PNN) [20], Deep Neural Networks (DNN) [21], Deep Recurrent Neural Networks (DRNN) [22], Multivariate LSTMFCNs [23], to improve forecasting performance. The RNN model can be used in various forecasting tasks because it has flexibility in the network, but has weaknesses in long-term forecasting [24], simple PNN in implementation, but needs to improve performance in testing so that it is more appropriate to apply in datasets that do not require training. Generative Adversarial Networks (GAN) is one of the training model frameworks to produce seemingly realistic data. The success of the GAN model in training two networks simultaneously makes this model accurate to use in the problem of detection of anomaly [26], [27], medical time series that have real value using conditional [28], Stock Market [29], portfolio management [30], [31], stock price manipulation detection [32], Estimated Renewable Energy Scenario [33], [34].

In this paper, we propose the GAN model for forecasting drug sales using generators and discriminators GAN trained on drug sales history data. GAN model training is trained in drug sales history data, each stock data is normalized, a time period of six days is constructed as input for GAN. After the GAN has finished training, the activated weight of the last layer puts the convolution used as a new representation of the data. Testing Data held in the training phase is run through the discriminator GAN section and the activated weights of the last convolutional layer are extracted. The extracted features are then classified to get predictive results. The model classification results will be evaluated with actual data indicators using evaluating Mean Absolute Error (MAE), Root Mean Square Error (RMSE) and Mean Absolute Percentage Error (MAPE). This paper is organized as follows: part 2 concerning related research, the method proposed is explained in section 3 , the results and discussion in section 4 and section 5 are conclusions.

\section{LITERATUR RIVIEW}

Time series forecasting is widely presented in the literature with applications in various fields such as care, health, inventory, climate modeling, financial trading and monitoring tools such as water, electricity and other quality [16], time series forecasting models not only focus on the results of predictive accuracy but must also measure and adjust to uncertainty over time. In depth learning (DN)models have demonstrated capabilities in the field of forecasting, such as financial forecasting in [17] using a combination of convolutional neural networks models with WaveNet architecture models to optimize access to historical data and optimize data processing and correlation structures between time, depth of air quality [36] proposing a hybrid model approach in one framework, and [37] proposing the RNN model for predicting proenvironment consumption status, predictive results evaluated by comparing the Artificial Neural Network model.

Research [38] proposes a deep neural network (DNN) method for estimating the consistent sales of pharmaceutical products in the next one week. Forecasting the scenario of a power plant process is presented by [35] which produces a series of scenarios that represent realistic possibilities for future behavior. Zhu, L., \& Laptev, N [39] reviewed models prediction number of trips during special events, driver incentive allocation and anomaly detection of requests. Forecasting electricity prices in [40], while Khashei, M., \& Hajirahimi, Z. [14], reviewed a combination of Hybrid ARIMA and multilayer perceptron neural network (MLP) to optimize stock market prediction results.

Some literature has established a GAN model framework approach improve the prediction of time series results such as, stock price forecasting reviewed [41], Chen, Y et all. [34] explored GANs with modeling uncertainty and variation in renewable energy that can be used as a decision-making tool in the electricity network, while [28] developed the GAN model to produce real-time multi-dimensional real-time series based on medical record data sourced from the patient's intensive care unit (ICU).

\section{PROPOSED METHOD}

In this section we describe the proposed method using the Generative Adversarial Networks (GAN) framework approach for forecasting sales data in hospitals. GAN is introduced by Goodfellow [42] as 
deep learning model framework for capturing the distribution of training data by generating new data from the same distribution using generator and discriminator models. Architecture GAN learns unsupervised features with a competitive learning process. GAN will produce more feature space that may be exploited, thereby reducing the potential for excess features during training. The following is an overview of the GAN model architecture that we are proposing in Figure 1.

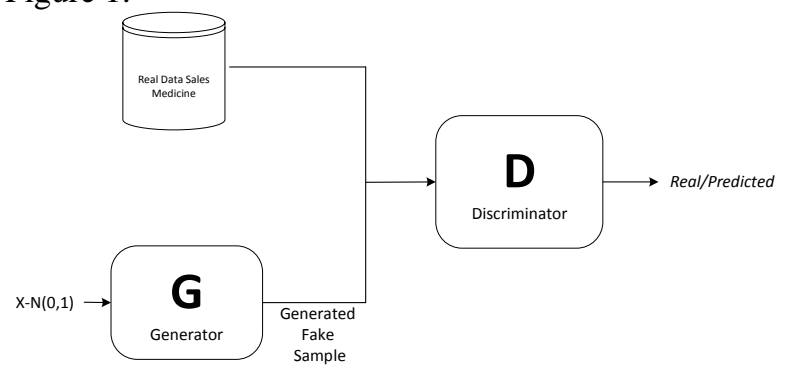

Figure 1 The proposed architecture method

The $\mathrm{G}$ (Generator) model is trained to produce data that looks like sales data from the target stock, while the D model (Discriminator) is trained to tell the difference between data from the Generator and real data. Errors from $\mathrm{D}$ are used to train $\mathrm{G}$ to defeat $\mathrm{D}$. The competition between $\mathrm{G}$ and $\mathrm{D}$ forces $\mathrm{D}$ to randomly distinguish from real variability, formally GAN solves the min-max game with the following equation:

$\max _{D} V(D)=\mathbb{E}_{\boldsymbol{x} \sim p_{\text {dat }}(\boldsymbol{x})}[\log D(\boldsymbol{x})]+\mathbb{E}_{\boldsymbol{z} \sim p_{\boldsymbol{z}}(\boldsymbol{z})}[\log (1-D(G(\boldsymbol{z})))$

Discriminator issues a $\mathrm{D}(\mathrm{x})$ value that indicates the possibility that $\mathrm{x}$ is real data with the aim of maximizing the opportunity to recognize real data as real data generated as false data. To measure the loss of the GAN model using cross-entropy, $p \log (q)$, for real data $p=1$. On the side of the generator, the objective function uses a model to produce the highest possible D

(x) value to overturn $\min _{G} V(G)=\mathbb{E}_{\boldsymbol{z} \sim p_{\boldsymbol{z}}(\boldsymbol{z})}[\log (1-D(G(\boldsymbol{z})))] \quad$ the difference.

As explained in the Goodfellow paper, D and $G$ play the min-max game where $D$ tries to maximize the probability of correctly classifying real and false $(\log D$ $(\mathrm{x})$ ), and $\mathrm{G}$ tries to minimize the probability that $\mathrm{D}$ will predict false output $(\log (1-\mathrm{D}(\mathrm{G}(\mathrm{x})))))$.

$\min _{G} \max _{D} V(D, G)=\mathbb{E}_{\boldsymbol{x} \sim p_{\text {data }}(\boldsymbol{x})}[\log D(\boldsymbol{x})]+\mathbb{E}_{\boldsymbol{z} \sim p_{\boldsymbol{z}}(\boldsymbol{z})}[\log (1-D(G(\boldsymbol{z})))]$

where $\mathrm{P}_{\text {data }}$ is the distribution of real training data from to $\mathrm{x}$, and $\mathrm{Pz}$ is the distribution of the $\mathrm{z}$ noise vector taken. $G$ is a mapping from $z$ to space $x$, whereas $D$ maps input $\mathrm{x}$ to a scalar value representing probabilistic $\mathrm{x}$ being the real sample.

\section{RESULT AND DISCUSSION}

\section{IV.1 Dataset Description}

In this section an explanation of the results of implementing the GAN model for forecasting drug sales, as the main purpose of this paper is to build a model that can forecast drug sales a week (7th day) in future, for this purpose, we use stock card record data drugs use consisting of 20 tables. This data contains 58,819 patients from 82 care units with a record of medicinuse transactions totaling 163,739 for inpatients and 262,140 for outpatients. From this data, we divide into three datasets, namely drug data (Drug Code, Drug Name, Type, Unit), sales data (Date, Qty, Drug Code, ICD Code, Sales Price) and purchase data (Date, Drug Code, Qty, Purchase Price) with focuses on seven forecasting indicator variables, namely the transaction date, drug code, ICD Code, sales price, purchase price, usage amount and total drug purchase.

Table 1 drug data

\begin{tabular}{|l|l|l|l|}
\hline Drug Code & Medicine Name & Type & Unit \\
\hline 13585 & FOLIC ACID 5 MG TAB & Obat & tab \\
\hline 13586 & DEXTAMINE SIRUP 60 ML & Obat & botol \\
\hline 13599 & SPEDIFEN 400 MG TABLET & Obat & tablet \\
\hline 13589 & SIMVASTATIN 20 MG TABLET & Obat & tablet \\
\hline 13588 & SOFTAMAN 1 LITER & Obat & botol \\
\hline 13580 & GLUCOPHAGE XR 500 MG TABLET & Obat & tablet \\
\hline 13596 & OXALIPLATIN 50 MG INJEKSI & Obat & vial \\
\hline.. &.. &.. &.. \\
\hline.. &.. &.. &.. \\
\hline 13601 & CALLUSOL 10 ML & Obat & botol \\
\hline
\end{tabular}

Table 2 Sales Data

\begin{tabular}{|c|c|c|c|c|}
\hline Date & Drug Code & ICD Code & Qty & Sales Price \\
\hline $27 / 05 / 201510: 58$ & 11858 & A00.9 & 20 & 1000 \\
\hline 07/01/2015 19:40 & 11966 & A02.2 & 1 & 1320 \\
\hline 07/01/2015 19:40 & 12370 & A00.1 & 1 & 2000 \\
\hline 07/01/2015 19:40 & 13068 & A02.2 & 1 & 100000 \\
\hline 07/01/2015 19:40 & 13144 & A05.8 & 2 & 5700 \\
\hline 07/01/2015 19:40 & 1455 & A09 & 10 & 5100 \\
\hline $17 / 02 / 201518: 35$ & 13293 & A05.8 & 1 & 25120 \\
\hline $17 / 02 / 2015$ 18:35 & 12862 & A16.9 & 3 & 1400 \\
\hline $17 / 02 / 201518: 35$ & 13317 & A17 & 3 & 7000 \\
\hline .. &.. & .. & .. & .. \\
\hline .. & .. & .. & .. & .. \\
\hline $17 / 02 / 201518: 35$ & 75 & A09 & 3 & 2000 \\
\hline
\end{tabular}

Table 3 Purchase Data

\begin{tabular}{|c|c|c|c|}
\hline Date & Drug Code & Purchase & Qty \\
\hline 05/01/2015 08:28 & 13862 & 45000 & 75 \\
\hline 05/01/2015 08:31 & 12619 & 51000 & 35 \\
\hline 05/01/2015 08:31 & 13862 & 45000 & 45 \\
\hline 05/01/2015 08:33 & 13875 & 2000 & 120 \\
\hline 05/01/2015 08:40 & 13862 & 45000 & 80 \\
\hline $05 / 01 / 201508: 43$ & 13780 & 90500 & 30 \\
\hline $05 / 01 / 201508: 48$ & 13045 & 353000 & 25 \\
\hline 05/01/2015 08:48 & 13183 & 4900 & 230 \\
\hline 05/01/2015 09:53 & 12728 & 6500 & 200 \\
\hline 05/01/2015 09:53 & 13753 & 9500 & 50 \\
\hline .. & .. &.. & .. \\
\hline $31 / 12 / 2015$ 10:31 & 10061 & 881.1 & 230 \\
\hline
\end{tabular}

Normalizing the required data as a key point to achieve competitive results [41], we normalize the data using the following equation: 


$$
x_{i} \frac{x_{i}-\mu^{t}}{\tau^{t}}
$$

Where $\mu^{t}$ and $\tau^{t}$ is the average standard deviation of X. we calculate the average standard deviation of 6 days to normalize the data, then the normalized data is used to predict data on day 7. Training and testing are processed simultaneously.

\section{IV.2 Model Training and Testing}

Our goal is to predict six factors and get the stock of supplies needed to optimize the planning of drug purchases in the next one week through historical data on medicine use records. Data is separated into two parts for training and testing models. We chose the data for the last 4 years (2015-2018) as training data, and data 2019 for testing. The time period was used as input for GAN. Each weight of GAN training activated in the last layer used representation of discriminator features to generation distribution as a new representation of data. The results of the GAN model training are shown in Figure 2 and Figure 3.

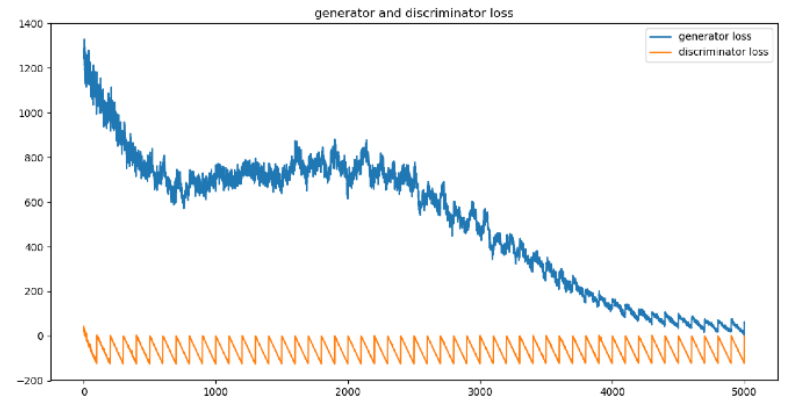

Figure 2 generator and discriminator

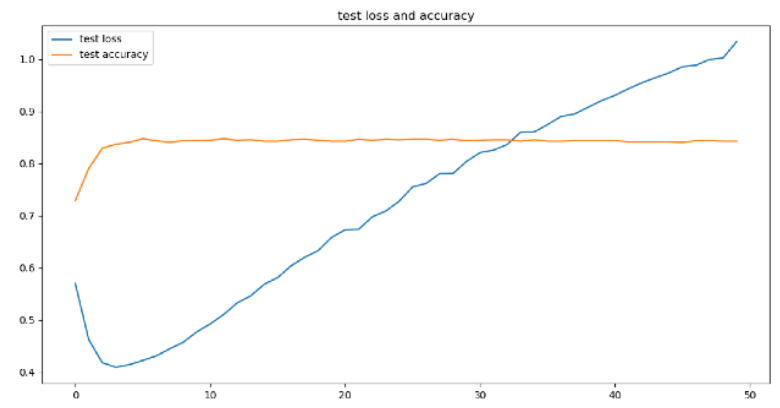

Figure 3 test lost and accuracy

Data testing in the training phase was carried out through GAN discriminator and weights from the last convolutional layer were extracted. The extracted features are then classified to measure the predicted results presented in Figure 4 and 5 images of the results of the coefficient matrix.

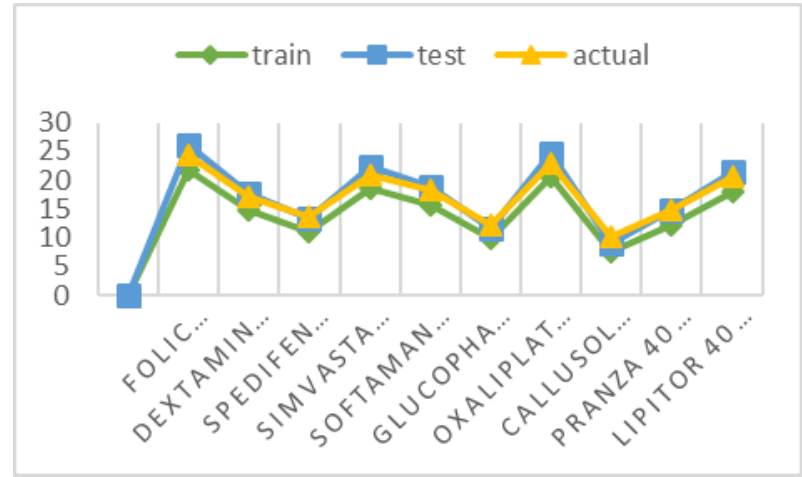

Figure 4 Prediction results of 10 types of drugs

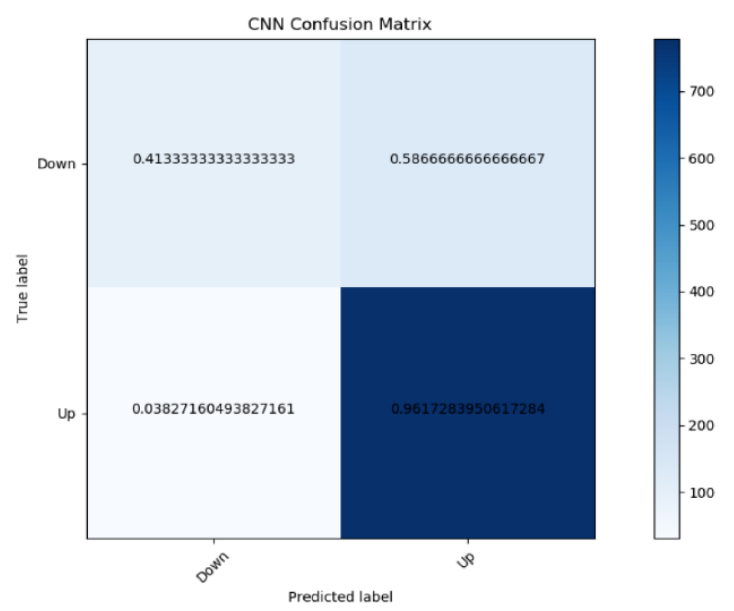

Figure 4 coefficient matrix Prediction results

The Matrix results show the results of the classification of the model. The perfect matrix will only have predictions on the main diagonal. The forecasting performance evaluation model that we propose uses statistical indicators, namely Mean Absolute Error (MAE), Root Mean Square Error (RMSE) and Mean Absolute Percentage Error (MAPE).

$$
\begin{aligned}
\text { MAE } & =\frac{1}{n} \sum_{j=1}^{n}\left|y_{j}-\hat{y}_{j}\right| \\
\text { RMSE } & =\sqrt{\frac{1}{n} \sum_{j=1}^{n}\left(y_{j}-\hat{y}_{j}\right)^{2}} \\
\text { MAPE } & =\frac{\sum \frac{|\mathrm{A}-\mathrm{F}|}{\mathrm{A}} \times 100}{\mathrm{~N}}
\end{aligned}
$$

Prediction testing based on the confusion matrix on the dataset will be calculated on average using RMSE, MAE and MAPE. The in table 5 which show result of the estimation with each value of epoch 100, 1000 and 5000. From the picture we can see that the proposed model has good performance according to the values of RMSE, MAE and MAPE, but overall it still needs to be developed understand the trend of use medicine. 
Table 4 The average evaluation on stock data sets

\begin{tabular}{|l|l|l|l|}
\hline Epoch & MAE & RMSE & MAPE \\
\hline 100 & 3.0901 & 5.1201 & 1.9201 \\
\hline 1000 & 2.1256 & 3.012 & 0.8034 \\
\hline 5000 & 1.0923 & 2.1093 & 0.0341 \\
\hline
\end{tabular}

\section{DISCUSSION AND SUGGESTION}

In this paper, we conduct exploration in forecasting the amount of drug sales over the one next week by trying to capture the distribution of drug use records according to actual data on the GAN framework that we propose, from the test results, the proposed model performs well in terms of MAE evaluation values, RMSE and MAPE, however it still needs to be developed and we plan to explore ways extract more valuable and influential factors from planning drug needs and optimizing our model in studying data distribution more accurately, so that proposed model obtains trend precision or predictions of higher drug requirements according needs

\section{ACKNOWLEDMENT}

This work is supported by Universitas Prima Indonesia, Fakultas Teknologi dan Ilmu Komputer, Program Studi Teknik Informatika.

\section{REFERENCES}

[1]. Husein, A. M., Harahap, M., Aisyah, S., Purba, W., \& Muhazir, A. (2018). The implementation of two stages clustering (k-means clustering and adaptive neuro fuzzy inference system) for prediction of medicine need based on medical data. In Journal of Physics: Conference Series (Vol. 978, No. 1, p. 012019). IOP Publishing.

[2]. Harahap, M., Husein, A. M., Aisyah, S., Lubis, F. R., \& Wijaya, B. A. (2018). Mining association rule based on the diseases population for recommendation of medicine need. In Journal of Physics: Conference Series (Vol. 1007, No. 1, p. 012017). IOP Publishing.

[3]. Borovykh, A., \& Bohte, S. (2017). Conditional Time Series Forecasting with Convolutional Neural Networks, 1-19.

[4]. Bao W, Yue J, Rao Y (2017) A deep learning framework for financial time series using stacked autoencoders and long-short term memory. PLoS ONE 12(7): e0180944. https://doi. org/10.1371/journal.pone.0180944
[5]. Ömer Faruk, D. (2010). A hybrid neural network and ARIMA model for water quality time series prediction. Engineering Applications of Artificial Intelligence, 23(4), 586-594. https://doi.org/10.1016/j.engappai.2009.09.015

[6]. Maestre, J. M., Fernández, M. I., \& Jurado, I. (2018). An application of economic model predictive control to inventory management in hospitals. Control Engineering Practice, 71(November 2017), 120-128. https://doi.org/10.1016/j.conengprac.2017.10.012

[7]. Ribeiro, A., Seruca, I., \& Durão, N. (2017). Improving organizational decision support: Detection of outliers and sales prediction for a pharmaceutical distribution company. Procedia Computer Science, 121, 282-290. https://doi.org/10.1016/j.procs.2017.11.039

[8]. Shin, J. S., Kim, S., \& Lee, J. M. (2015). Production and inventory control of auto parts based on predicted probabilistic distribution of inventory. Digital Communications and Networks, $1(4)$, 292-301. https://doi.org/10.1016/j.dcan.2015.10.002

[9]. De Santis, R. B., De Aguiar, E. P., \& Goliatt, L. (2018). Predicting material backorders in inventory management using machine learning. 2017 IEEE Latin American Conference on Computational Intelligence, LA-CCI 2017 Proceedings, 2017-November, 1-6. https://doi.org/10.1109/LA-CCI.2017.8285684

[10]. Bozkir, A. S., \& Sezer, E. A. (2011). Predicting food demand in food courts by decision tree approaches. Procedia Computer Science, 3, 759763. https://doi.org/10.1016/j.procs.2010.12.125

[11]. De Marcos, R. A., Bello, A., \& Reneses, J. (2017). Short-term forecasting of electricity prices with a computationally efficient hybrid approach. International Conference on the European Energy Market, EEM, 6-11. https://doi.org/10.1109/EEM.2017.7981946

[12]. Beigaite, R., \& Krilavičius, T. (2018). Electricity price forecasting for nord pool data using recurrent neural networks. CEUR Workshop Proceedings, 2145, 75-78. https://doi.org/10.3390/en11051255

[13]. Ugurlu, U. (2018). Electricity Price Forecasting Using Recurrent Neural Networks, (April), 1-21. https://doi.org/10.3390/en11051255

[14]. Khashei, M., \& Hajirahimi, Z. (2018). A comparative study of series arima/mlp hybrid models for stock price forecasting. Communications in Statistics: Simulation and 
Computation, $\quad 0(0), \quad 1-16$. https://doi.org/10.1080/03610918.2018.1458138

[15]. Angamuthu Chinnathambi, R., Mukherjee, A., Campion, M., Salehfar, H., Hansen, T., Lin, J., \& Ranganathan, P. (2018). A Multi-Stage Price Forecasting Model for Day-Ahead Electricity Markets. $\quad$ Forecasting, 1(1), 3. https://doi.org/10.3390/forecast1010003

[16]. Orozco, B. P., Abbati, G., \& Roberts, S. (2018). MOrdReD: Memory-based Ordinal Regression Deep Neural Networks for Time Series Forecasting. Retrieved from http://arxiv.org/abs/1803.09704

[17]. Borovykh, A., Bohte, S., \& Oosterlee, C. W. (2017). Conditional time series forecasting with convolutional neural networks. Lecture Notes in Computer Science (Including Subseries Lecture Notes in Artificial Intelligence and Lecture Notes in Bioinformatics), 10614 LNCS, 729-730. https://doi.org/10.1007/978-3-319-68612-7

[18]. Graves, A. (2016). Adaptive Computation Time for Recurrent Neural Networks, 1-19. https://doi.org/10.475/123

[19]. Bianchi, F. M., Maiorino, E., Kampffmeyer, M. C., Rizzi, A., \& Jenssen, R. (2017). An overview and comparative analysis of Recurrent Neural Networks for Short Term Load Forecasting, 1-41. https://doi.org/10.1007/978-3-319-70338-1

[20]. Qu, Y., Cai, H., Ren, K., Zhang, W., Yu, Y., Wen, Y., \& Wang, J. (2017). Product-based neural networks for user response prediction. Proceedings - IEEE International Conference on Data Mining, ICDM, 1149-1154. https://doi.org/10.1109/ICDM.2016.57

[21]. Zhang, L., Wang, G., \& Giannakis, G. B. (2018). Real-time Power System State Estimation and Forecasting via Deep Neural Networks, 1-9. Retrieved from http://arxiv.org/abs/1811.06146

[22]. Prasad, S. C., \& Prasad, P. (2014). Deep Recurrent Neural Networks for Time- Series Prediction. 1407.5949, 95070, 1-19.

[23]. Karim, F., Majumdar, S., Darabi, H., \& Harford, S. (2018). Multivariate LSTM-FCNs for Time Series Classification, 1-9. Retrieved from http://arxiv.org/abs/1801.04503

[24]. Hsu, D. (2017). Multi-period Time Series Modeling with Sparsity via Bayesian Variational Inference, 1-14. Retrieved from http://arxiv.org/abs/1707.00666

[25]. Müller-Navarra, M., Lessmann, S., \& Voß, S. (2015). Sales forecasting with partial recurrent neural networks: Empirical insights and benchmarking results. Proceedings of the Annual Hawaii International Conference on System Sciences, 2015-March, 1108-1116. https://doi.org/10.1109/HICSS.2015.135

[26]. Li, D., Chen, D., Shi, L., Jin, B., Goh, J., \& Ng, S.-K. (2019). MAD-GAN: Multivariate Anomaly Detection for Time Series Data with Generative Adversarial Networks, 1-17. https://doi.org/arXiv:1809.04758v1

[27]. Li, D., Chen, D., Goh, J., \& Ng, S. (2018). Anomaly Detection with Generative Adversarial Networks for Multivariate Time Series, 1-10. https://doi.org/arXiv:1809.04758v1

[28]. Esteban, C., Hyland, S. L., \& Rätsch, G. (2017). Real-valued (Medical) Time Series Generation with Recurrent Conditional GANs. https://doi.org/10.1002/fut

[29]. Zhou, X., Pan, Z., Hu, G., Tang, S., \& Zhao, C. (2018). Stock Market Prediction on HighFrequency Data Using, 2018.

[30]. Liang, Z., Jiang, K., Chen, H., Zhu, J., \& Li, Y. (2018). Deep Reinforcement Learning in Portfolio Management. https://doi.org/arXiv:1808.09940v2

[31]. Liang, Z., Chen, H., Zhu, J., Jiang, K., \& Li, Y. (2018). Adversarial Deep Reinforcement Learning in Portfolio Management. https://doi.org/10.1111/j.1476-5829.2011.00268.X

[32]. Leangarun, T., Tangamchit, P., \& Thajchayapong, S. (2018). Stock Price Manipulation Detection using Generative Adversarial Networks. In 2018 IEEE Symposium Series on Computational Intelligence (SSCI) (pp. 2104-2111). IEEE

[33]. Chen, Y., Li, P., \& Zhang, B. (2018). Bayesian renewables scenario generation via deep generative networks. 2018 52nd Annual Conference on Information Sciences and Systems, CISS 2018, 1-6. https://doi.org/10.1109/CISS.2018.8362314

[34]. Chen, Y., Wang, Y., Kirschen, D., \& Zhang, B. (2018). Model-Free Renewable Scenario Generation Using Generative Adversarial Networks. IEEE Transactions on Power Systems, 33(3), 3265-3275. https://doi.org/10.1109/TPWRS.2018.2794541

[35]. Borovykh, A., Bohte, S., \& Oosterlee, C. W. (2017). Conditional time series forecasting with convolutional neural networks. Lecture Notes in Computer Science (Including Subseries Lecture Notes in Artificial Intelligence and Lecture Notes in Bioinformatics), 10614 LNCS, 729-730. https://doi.org/10.1007/978-3-319-68612-7 
[36]. Du, S., Li, T., Yang, Y., \& Horng, S. J. (2018). Deep Air Quality Forecasting Using Hybrid Deep Learning Framework. arXiv preprint arXiv:1812.04783.

[37]. Lee, D. (2017). Using Deep Learning Techniques to Forecast Environmental Consumption Level, 117. https://doi.org/10.3390/su9101894

[38]. Tech, C. Y., Networks, N., Detection, O. N., Digital, F., View, I., \& Network, G. N. (2017). A Deep Learning Algorithm to Forecast Sales of Pharmaceutical Products A Deep Learning Algorithm to Forecast Sales of Pharmaceutical Products, (August).

[39]. Zhu, L., \& Laptev, N. (2017). Deep and Confident Prediction for Time Series at Uber. IEEE International Conference on Data Mining Workshops, ICDMW, 2017-Novem, 103-110. https://doi.org/10.1109/ICDMW.2017.19

[40]. Kuo, P. H., \& Huang, C. J. (2018). An electricity price forecasting model by hybrid structured deep neural networks. Sustainability (Switzerland), 10(4), 1-17. https://doi.org/10.3390/su10041280

[41]. Zhang, K., Zhong, G., Dong, J., Wang, S., \& Wang, Y. (2019). Stock Market Prediction Based on Generative Adversarial Network. Procedia Computer Science, 147, 400-406. https://doi.org/10.1016/J.PROCS.2019.01.256

[42]. Goodfellow, I. J., Pouget-Abadie, J., Mirza, M., Xu, B., Warde-Farley, D., Ozair, S., ... Bengio, Y. (2014). Generative Adversarial Networks, 1-9. https://doi.org/10.1017/CBO978113905845 